\title{
In vivo osteogenic activity of bone marrow stromal stem cells transfected with Ad-GFP-hBMP-2
}

\author{
G.X. Wang ${ }^{1 *}$, L. Hu ${ }^{3 *}$, H.X. Hu ${ }^{2}$, Z. Zhang ${ }^{2}$ and D.P. Liu ${ }^{2}$ \\ ${ }^{1}$ Department of Pharmacology, Liaoning Medical University, Jinzhou, China \\ ${ }^{2}$ Department of Orthopaedic Surgery, \\ First Affiliated Hospital of Liaoning Medical University, Jinzhou, China \\ ${ }^{3}$ Department of Orthopaedics, Jinmen No. 1 People's Hospital, Jinmen, China \\ *These authors contributed equally to this study. \\ Corresponding author: D.P. Liu \\ E-mail: yingmingyangcn@163.com
}

Genet. Mol. Res. 13 (2): 4456-4465 (2014)

Received May 14, 2013

Accepted December 4, 2013

Published June 16, 2014

DOI http://dx.doi.org/10.4238/2014.June.16.4

\begin{abstract}
The aim of this study was to investigate the expression of bone morphogenetic protein-2 (BMP-2) in bone marrow stromal stem cells (BMSCs) and the in vivo and in vitro osteogenic activity of BMSCs transfected with the adenovirus plasmid, Ad-GFP-hBMP-2. The Ad-GFP-hBMP-2 plasmid was packaged and transfected into rabbit BMSCs to determine the transfection rate. The alkaline phosphatase (ALP) activities of Ad-GFP-hBMP-2-transfected BMSCs (experimental group) and untransfected BMSCs (control group) were detected. In situ hybridization of type I collagen and Western blot were used to determine the BMP-2 gene and protein expressions. The transfected and untransfected BMSCs were respectively inoculated into nude mice to observe in vivo osteogenesis. The decalcified bovine cancellous bone scaffold was respectively combined with transfected and untransfected BMSCs and implanted into ulnar defects in rabbits to repair the bone. The adenovirus titer was $1.2 \times 10^{10} \mathrm{pfu} / \mathrm{mL}$. Green fluorescent protein expression appeared $48 \mathrm{~h}$ after transfection with the adenovirus plasmid, and the transfection rate was $71.1 \%$. The ALP activity was higher in
\end{abstract}


the experimental group than the control group at each time point after transfection. The gene and protein expressions of BMP-2 were higher in the experimental group than the control group. The positive rates of in vivo osteogenesis in the experimental and control groups were $90 \%$ and $40 \%$, respectively. The bone defect repair effects differed markedly between the two groups. The BMP-2 gene can be highly expressed in BMSCs to successfully induce osteogenic differentiation. BMSCs can be used as seed cells for bone tissue engineering.

Key words: Bone morphogenetic protein; Bone marrow stromal stem cells; Bone tissue engineering

\section{INTRODUCTION}

Bone defect repair is a difficult problem in the medical field, and progress in tissue engineering technology has provided a new way for solving this problem (Xynos et al., 2000; Pérez-Sánchez et al., 2010; Amini et al., 2012), in which seed cells, carrier material, and growth factors are essential (Liu et al., 2010a). Ideal seed cells should have the following characteristics: 1) easy to obtain with minimal injury to the body; 2) high proliferation ability in in vitro culture, with easy and stable expression into the osteoblast phenotype; 3 ) tolerance to body immunity, with ability of continued and rapid osteogenesis; 4) no tumorigenicity (Kumar et al., 2010; Liu et al., 2010b; Neman et al., 2012a). Bone marrow stromal cells (BMSCs) have these advantages and have therefore received more and more research attention recently (Porter et al., 2009). The differentiation of seed cells relies on the surrounding microenvironment. Many growth factors can induce the differentiation of BMSCs into osteoblasts, of which bone morphogenetic protein (BMP) has been shown to have a clear osteogenesis inducing effect (Meinel et al., 2006; Menendez et al., 2011). However, individual BMP molecules will rapidly diffuse after implantation in the body, are easily degraded by proteases, and cannot effectively act on more target cells in an efficient manner. Therefore, the osteogenesis inducing activity of BMP cannot be fully exerted, which greatly limits its clinical application. In tissue engineering technology, the exogenous BMP-2 gene is transfected into seed cells to express BMP-2, which induces the differentiation of seed cells into osteoblasts. This method can overcome the various disadvantages of the in vivo growth of exogenous growth factors, and has become a new treatment strategy. In this study, using adenovirus as a mediator, the BMP-2 and green fluorescent protein (GFP) genes were transfected into BMSCs, and the feasibility of constructing tissueengineered bone with BMSCs transfected by this Ad-GFP-hBMP-2 plasmid was investigated.

\section{MATERIAL AND METHODS}

\section{Adenovirus packaging}

The ultra-pure plasmid Ad-GFP-hBMP-2 (previously prepared in the Central Laboratory of the First Affiliated Hospital of Liaoning Medical University) was digested with $\mathrm{PacI}$ restriction enzyme, and the large fragments were extracted and purified. Under the mediation of Lipofectamine 2000 (Invitrogen Corp., USA), they were transfected into HEK293A 
cells (previously prepared in the Central Laboratory of the First Affiliated Hospital of Liaoning Medical University) for 7 days of packaging. The transfection effect was observed. After freezing and thawing 3 times, the adenovirus solution was obtained, and the virus titer was detected using the plaque assay, followed by preservation at $-80^{\circ} \mathrm{C}$ until subsequent use.

\section{Transfection}

Four milliliters bone marrow were drawn from the right tibial plateau of clean grade, 2-3 month-old Japanese white rabbits weighing $1.9 \mathrm{~kg}$, which were provided by the Experimental Animal Center of Liaoning Medical University. After separation, purification, and culture, third generation BMSCs were obtained and were transfected with the adenovirus solution described above, with a multiplicity of infection (MOI) of 100. The transfection effect was observed under an inverted fluorescence microscope.

\section{Transfection rate}

Single cell suspensions of $10^{6}$ cells/mL Ad-GFP-hBMP-2-transfected BMSCs were prepared. After removal of cell clusters by filtration with a 150-mesh gauze, flow cytometry was performed with a $488 \mathrm{~nm}$ excitation light and a $530 \mathrm{~nm}$ receiving light. The results were analyzed using the Cellquest software, and are represented as the transfection rate (percentage of GFP-positive cells) and the mean fluorescence intensity of GFP-positive cells.

\section{Alkaline phosphatase (ALP) activity}

Ad-GFP-hBMP-2-transfected and untransfected BMSCs (experimental and control groups, respectively) were inoculated into 96 -well plates, respectively, at a 2000 cells/ well density. After cell adherence, BMSCs were transfected by adenovirus solution (MOI $=100$ ) followed by routine culture. At the $3 \mathrm{rd}, 6 \mathrm{th}, 9 \mathrm{th}$, and 12th days after transfection, the supernatant of each well was removed, followed by washing with phosphate-buffered saline (PBS). Then, $100 \mu \mathrm{L} 0.1 \%$ Triton 100 was added to each well for overnight cleavage at $4{ }^{\circ} \mathrm{C}$. The ALP activity was detected using an ALP kit (Upstate Biotechnology Inc.; Massachusetts, USA) according to manufacturer instructions. Using the continuous monitoring method for $3 \mathrm{~min}$, the absorbance change per minute $(\Delta \mathrm{A} / \mathrm{min})$ was detected at a $405 \mathrm{~nm}$ wavelength. The actual concentration of ALP was calculated according to the formula: $\operatorname{ALP}(\mathrm{U} / \mathrm{L})=\Delta \mathrm{A} / \min \times 2757$.

\section{BMP-2 gene expression}

Expression of the BMP-2 gene in Ad-GFP-hBMP-2-transfected and untransfected BMSCs were determined by in situ hybridization of type I collagen. The slide was placed in a 2-well plate, and $1 \times 10^{4}$ BMSCs were added to each well. After cell adherence, BMSCs were transfected with adenovirus solution $(\mathrm{MOI}=100)$, followed by routine culture for 2 weeks. The mRNA fragments were exposed, and in situ hybridization of type I collagen was conducted (Upstate Biotechnology Inc.), followed by slide mounting with neutral gum. In the control group, BMSCs were not transfected by Ad-GFP-hBMP-2. 


\section{Western blot}

Total protein of Ad-GFP-hBMP-2 transfected and untransfected BMSCs was extracted from the cell lysates, respectively, followed by determination of protein expression using polyacrylamide gel electrophoresis on 5\% stacking gel and $8 \%$ separation gel at $60 \mathrm{~V}$ for 30 min and $150 \mathrm{~V}$ for $1 \mathrm{~h}$. After electrophoresis, the separation gel was washed with PBS three times, followed by transmembrane $(100 \mathrm{~mA}$ for $30 \mathrm{~min})$. Then, primary antibody (1:1500; Santa Cruz Biotechnology, Inc., USA) was added, followed by incubation at $4{ }^{\circ} \mathrm{C}$ overnight. After washing the membrane, secondary antibody (Santa Cruz Biotechnology, Inc.) and developing solution were added, followed by incubation for $30 \mathrm{~min}$ at room temperature in the dark. The coloration was terminated by washing with PBS three times. The optical density (OD) values of internal reference target bands were analyzed using a gel imaging system. Each determination was repeated three times.

\section{Osteogenesis experiment}

The in vivo osteogenesis experiment was conducted using 24 nude mice 8 weeks of age with an average weight of $22.5 \mathrm{~g}$ from the Experimental Animal Center of China Medical University. This study was carried out in strict accordance with the recommendations in the Guide for the Care and Use of Laboratory Animals of the National Institutes of Health. The animal use protocol was reviewed and approved by the Institutional Animal Care and Use Committee (IACUC) of Liaoning Medical University. Suspensions of $2.5 \times 10^{7}$ cells $/ \mathrm{mL}$ Ad-GFP-hBMP2-transfected and untransfected BMSCs were prepared, respectively. The $5 \times 10^{6}$ cell suspension was injected into the posterior buttock muscle of mice (left side, experimental side; right side, control side). The animal response after injection was observed. At the 12th postoperative week, X-ray examination was performed. Then the nude mouse was executed, and bone tissue specimens were taken, followed by hematoxylin and eosin (HE) staining and observation.

\section{Bone defect repair experiment}

The decalcified bovine cancellous bone scaffold (Central Laboratory of the First Affiliated Hospital of Liaoning Medical University) was pretreated and dried. Suspensions of $2.5 \times 10^{7}$ cells $/ \mathrm{mL}$ Ad-GFP-hBMP-2-transfected and untransfected BMSCs were dripped into the scaffold at $5 \times 10^{6}$ cells/scaffold, followed by culture for 2 days. One-centimeter bone defects in the bilateral middle ulna were made in 24 Japanese white rabbits (left side, experimental side; right side, control side). On the experimental side in the experimental and control groups, the defect area was implanted with the scaffold with Ad-GFP-hBMP-2-transfected and untransfected BMSC complexes, respectively. The control sides in the two groups were not implanted with any material. The postoperative animal response was observed. At the 12 th postoperative week, X-ray examination on the ulna was performed. Then, the rabbits were executed, and the ulna specimens were taken, followed by HE staining and observation.

\section{Statistical analysis}

Statistical analysis was performed using the SPSS 17.0 statistical software. Measurement 
data are reported as means $\pm \mathrm{SD}$, and the Student $t$-test was used to analyze differences between the two groups. The chi-square test or the exact probability test was performed for comparing enumeration data between the two groups. $\mathrm{P}<0.05$ was considered to be statistically significant.

\section{RESULTS}

\section{Transfection}

After $48 \mathrm{~h}$ from transfection with the adenovirus plasmid, HEK293A cells began to detach from the wall. The green fluorescent cells were visible under fluorescence microscope. At the 7th day, all of the cells had detached. There were many green fluorescent cells in the full field of view, suggesting successful packaging of the adenovirus (Figure 1A). Results of the plaque assay showed that the titer of Ad-GFP-hBMP-2 was $1.2 \times 10^{10} \mathrm{PFU} / \mathrm{mL}$. At the 24th $\mathrm{h}$ after transfection, the fluorescent cells began to appear. At the 48th $\mathrm{h}$, the number of fluorescent cells increased significantly (Figure 1B). Results of flow cytometry showed that the percentage of GFP-positive cells was high, suggesting a high transfection rate of $71.1 \%$ (Figure 1C).
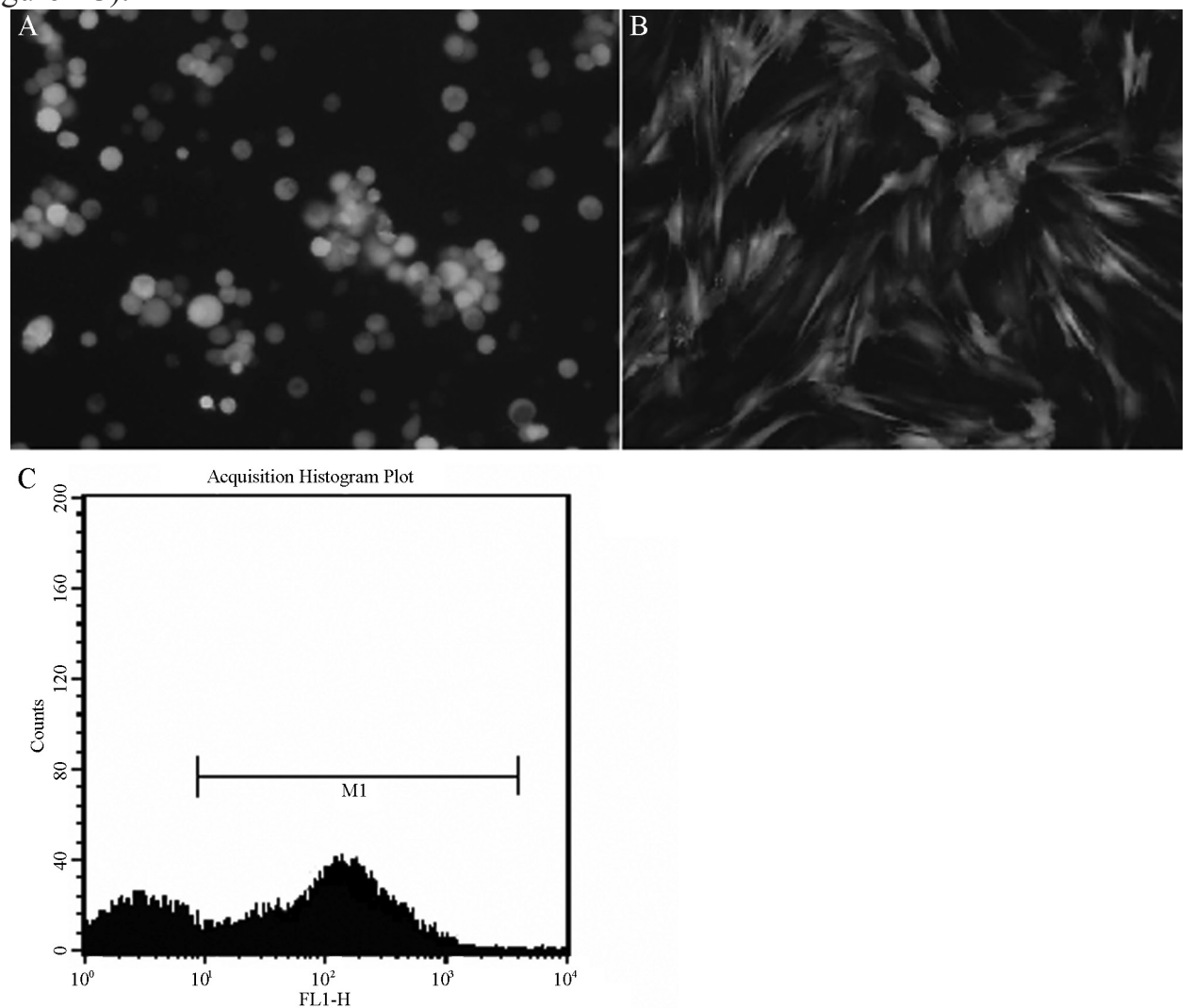

Figure 1. Transfection results. A. Observation of HEK293A cells after 7 days from transfection (100X). B. Observation of BMSCs $48 \mathrm{~h}$ after transfection (100X) C. Results of flow cytometry (M1 represents the transfection rate of $71.1 \%$ ). 


\section{ALP activity}

As shown in Table 1, the ALP activity in the experimental group and the control group gradually increased with increasing culture time, with significant differences observed among time points $(\mathrm{P}<0.01)$. At each time point, the ALP activity in the experimental group was

Table 1. ALP activity at each time point in two groups (U/L).

\begin{tabular}{lcccc}
\hline Group & 3rd day & 6th day & 9th day & 12th day \\
\hline Experimental & $4.33 \pm 3.51$ & $8.26 \pm 3.57$ & $16.54 \pm 6.75$ & $20.08 \pm 8.38$ \\
Control & $20.48 \pm 9.79$ & $48.05 \pm 9.40$ & $74.44 \pm 9.28$ & $86.65 \pm 8.70$ \\
$\mathrm{t}$ & 4.235 & 4.844 & 5.484 & 5.624 \\
$\mathrm{P}$ & $<0.001$ & $<0.001$ & $<0.001$ & $<0.001$ \\
\hline
\end{tabular}

higher than that in the control group $(\mathrm{P}<0.01)$.

\section{Western blot}

At the 12th day after transfection of Ad-GFP-hBMP-2 into BMSCs, in the experimental group, the in situ hybridization was positive, with many brown particles observed in the cytoplasm (Figure 2A). In the control group, the in situ hybridization was negative or only weakly positive, with less or no brown particles apparent in the cytoplasm

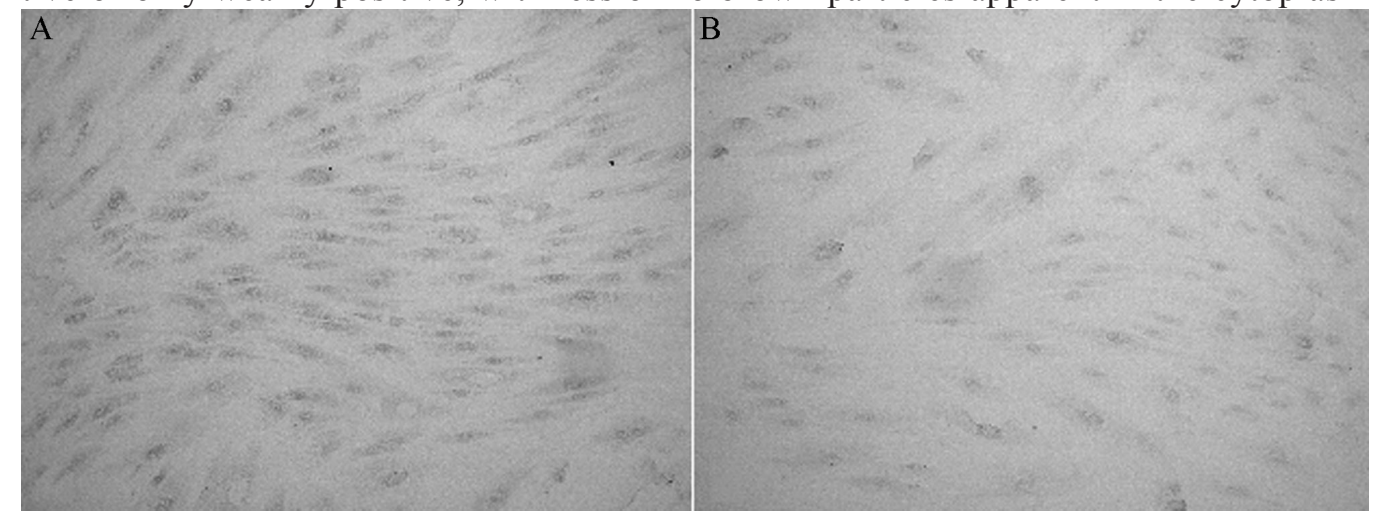

Figure 2. Results of in situ hybridization of type I collagen (100X).

(Figure 2B).

The Western blot results showed that at the 4th and 8th days after transfection, there was obvious BMP-2 expression in the experimental group, whereas there was no BMP-2 expression observed in the control group at these times (Figure 3).

\section{In vivo osteogenesis}


X-ray examination results showed that at the 12th week after implantation of BMSCs, on the experimental sides of the experimental group, 7, 2, and 1 sides showed highdensity shadows, slightly high-density shadows, and no high-density shadow, respectively; the positive rate was $90 \%$. In the control group, there were 4 sides with high-density shadows (uneven density), with the remaining 6 sides showing no high-density shadow; the positive rate was $40 \%$ (Figure 4A). Histological observation results showed that on the experimental sides of the experimental group, there was increased formation of mature bone tissue, with green fluorescence apparent under the fluorescence microscope. In the control group, there was partially immature bone tissue and cartilage tissue in the muscle Experimental group Control group

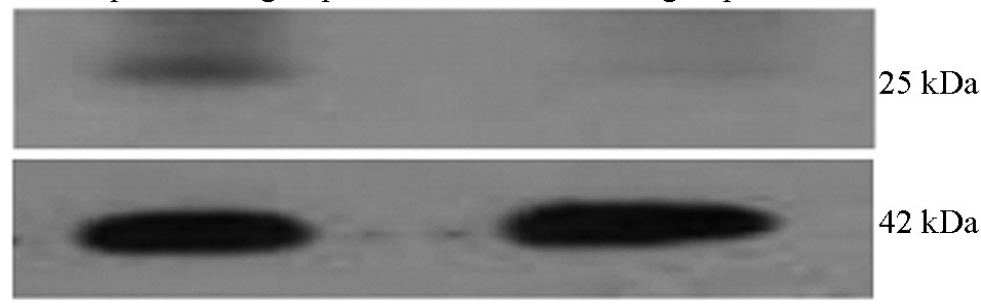

Figure 3. BMP-2 expressions in the two groups.

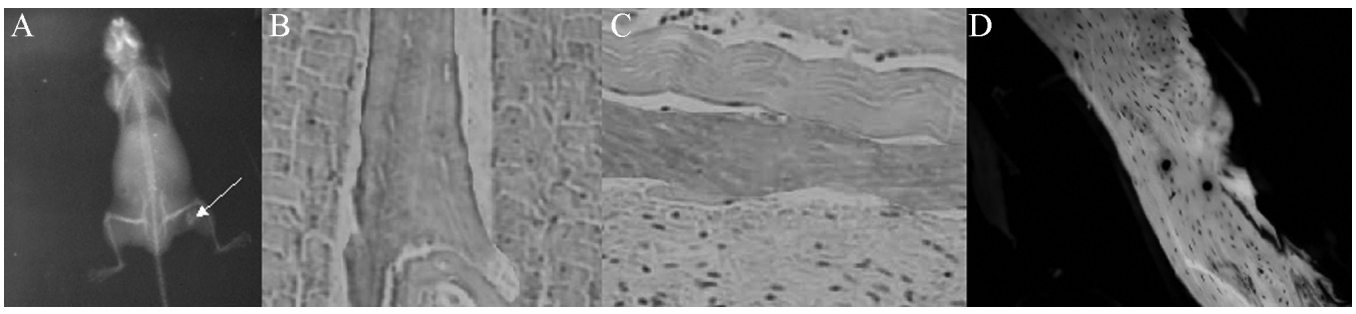

Figure 4. Results of in vivo osteogenesis. A. X-ray examination of the ulna (12 weeks). B. Histological observation of an ulna specimen on the experimental side of an animal of the experimental group (100X). C. Histological observation of an ulna specimen on the control side of an animal of the experimental group (100X). D. Fluorescent microscopy of an ulna specimen on the experimental side of an animal of the experimental group (100X).

tissue (Figure 4B-D).

\section{Bone defect repair}

At the 12th week after transplantation of the scaffold and BMSCs complex, the experimental group showed continuous bone callus formation on each experimental side. In the control group, 3 sides showed continuous bone callus formation and 3 sides had obvious fracture lines. The density of the defect area was higher on the experimental side than the control side (Figure 5A, B). Histological observation results showed increased bone tissue on the experimental side in the experimental group compared to the control group, with bulky bone trabecula. In the control group, the bone trabecula was slender with evident cartilage formation (Figure $5 \mathrm{C}, \mathrm{D}$ ). On the experimental sides of the experimental group, green fluorescence of the bone tissue was apparent under the fluorescence 


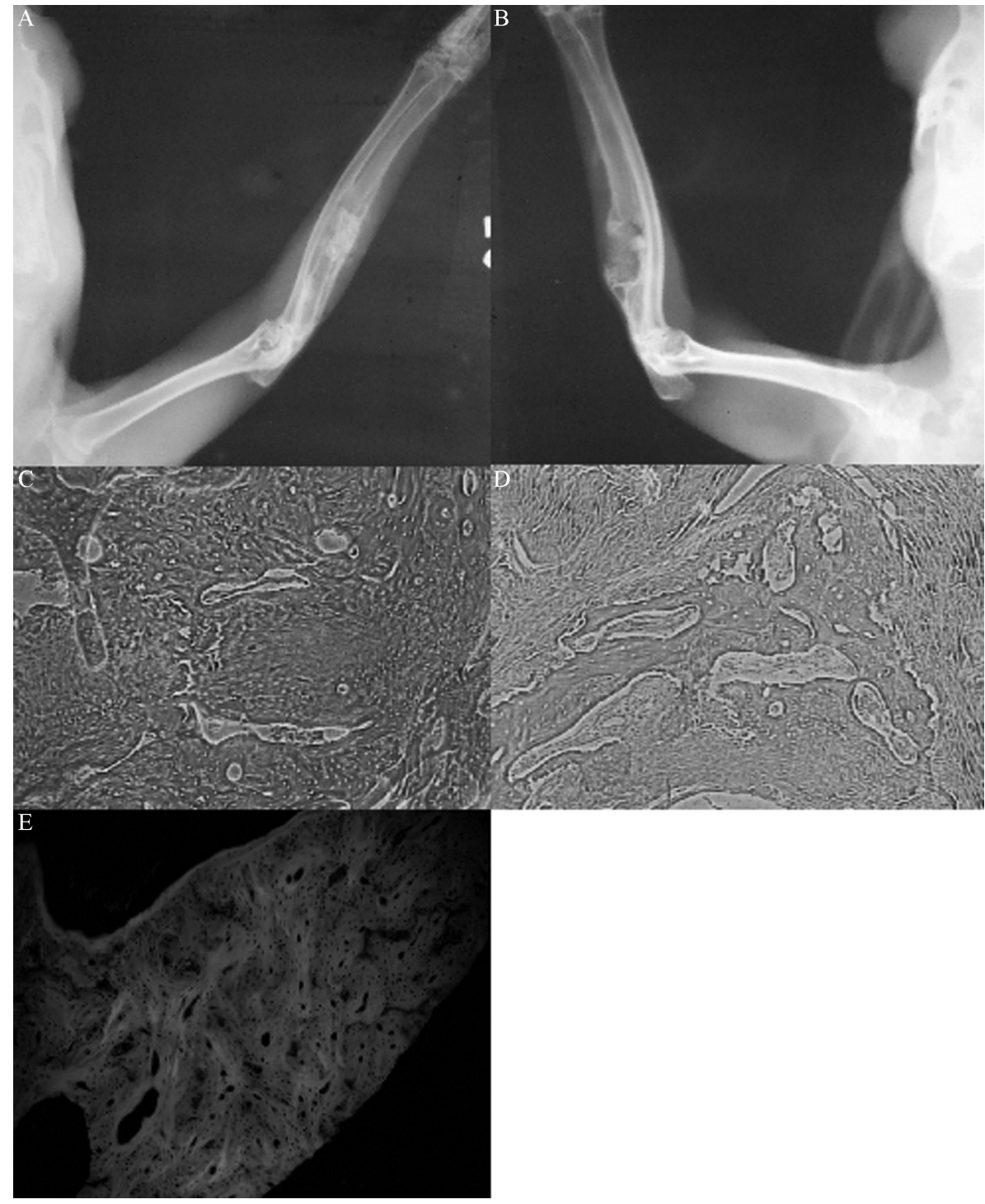

Figure 5. Results of bone defect repair. A. X-ray examination of the bone defect area on the experimental side of an animal of the experimental group (12 weeks). B. X-ray examination of the bone defect area on the experimental side of the control group (12 weeks). C. Histological observation of the bone defect area on the experimental side of the experimental group (12 weeks, 100X). D. Histological observation of the bone defect area on the experimental side of the control group (12 weeks, 100X). E. Fluorescent microscopy of the bone defect area on the experimental side of an animal of the experimental group (100X).

microscope (Figure 5E).

\section{DISCUSSION}


Seed cells are one of the three basic elements for bone tissue engineering (Neman et al., 2012b). BMSCs are stable, and are easily obtained. In addition, BMSCs have potential osteogenic differentiation characteristics, with little injury to the body. Therefore, they have been widely used in tissue engineering, and have become a hotspot in research of seed cells (Chang et al., 2009; Ciapetti et al., 2012; Kang et al., 2012). Due to their undifferentiated state, BMSCs have strong proliferative activity but weak osteogenic activity. They can only become true seed cells after induction. Transgenic engineering can achieve the expression of target genes and related growth factors that have such induction effects. In this study, using adenovirus as a vector, the BMP-2 gene was transfected into BMSCs. The objective was to verify the expression of BMP-2 in BMSCs, its in vitro and in vivo osteogenesis inducing effect, and to provide an experimental basis for the application of this new type of seed cell.

Recently, the transfection rate and cytotoxicity of adenovirus have received more and more attention. Increasing the MOI can not only improve the transfection rate, but also increase the cytotoxicity. In this study, according to results of our preliminary experiments and previous studies, the MOI was set at 100. This was sufficient to resolve the conflict between the transfection rate and cytotoxicity, and yielded a transfection rate of more than $70 \%$, which is in accordance with the results of a previous study (Liu et al., 2013).

In order to facilitate the detection of gene expression after transfection into seed cells and the in vivo osteogenic activity, the reporter molecule gene GFP is transfected into seed cells. Compared with using a single vector to carry reporter genes or target genes, the simultaneous transfection of two kinds of genes into seed cells provides a convenient way to detect genetically engineered seed cells in in vitro culture and in vivo osteogenesis (Misteli and Spector, 1997; Chen et al., 2009; Salunkhe et al., 2010). After transfection of Ad-GFP-hBMP-2 into BMSCs, the target gene and reporter gene could be expressed at the same time. The gene transfection and expression were determined by observing the GFP expression. The reliability of this molecular marker has been confirmed in animal experiments.

Due to congenital developmental defects and immature lymphocyte development, nude mice have no immunological competence. Therefore, they are widely used to construct models of tumor cell transplantation (Jin et al., 2010). The adenovirus used in this study is a type of modified adenovirus, which cannot escape the immune attack from the host. Application of nude mice can ensure the in vivo expression of the adenovirus-carried gene, without it being removed. In addition, whether the adenovirus has antigenicity in cells and whether the adenovirus skeleton gene itself can be expressed remain unclear. In this study, the adenovirus transfected BMSCs combined with decalcified bovine cancellous bone successfully repaired the long segment defect of rabbit ulnae, and the repair effect of the experimental group was better than that of the control group. This indicates that the antigenicity of seed cells transfected by the adenovirus is weak, and it did not affect the osteogenic activity. At the same time, this result also provides evidence for the potential role of decalcified bovine cancellous bone in bone tissue engineering. BMSCs have the characteristic of adherent growth, and the demineralized bovine cancellous bone can provide adherence support. The combination of BMSCs and decalcified bovine cancellous bone is a classic model for constructing tissue-engineered bone, and has provided a new method for bone defect repair.

\section{REFERENCES}


Amini AR, Laurencin CT and Nukavarapu SP (2012). Bone tissue engineering: recent advances and challenges. Crit. Rev. Biomed. Eng 40: 363-408.

Chang SC, Lin TM, Chung HY, Chen PK, et al. (2009). Large-scale bicortical skull bone regeneration using ex vivo replication-defective adenoviral-mediated bone morphogenetic protein-2 gene-transferred bone marrow stromal cells and composite biomaterials. Neurosurgery 65: 75-81.

Chen J, Sai SY, Vazin T, Coggiano M, et al. (2009). Human embryonic stem cells which express hrGFP in the undifferentiated state and during dopaminergic differentiation. Restor. Neurol. Neurosci. 27: 359-370.

Ciapetti G, Granchi D and Baldini N (2012). The combined use of mesenchymal stromal cells and scaffolds for bone repair. Curr. Pharm. Des. 18: 1796-1820.

Jin K, Teng L, Shen Y, He K, et al. (2010). Patient-derived human tumour tissue xenografts in immunodeficient mice: a systematic review. Clin. Transl. Oncol. 12: 473-480.

Kang SW, Bae JH, Park SA, Kim WD, et al. (2012). Combination therapy with BMP-2 and BMSCs enhances bone healing efficacy of PCL scaffold fabricated using the 3D plotting system in a large segmental defect model. Biotechnol. Lett. 34: 1375-1384.

Kumar S, Wan C, Ramaswamy G, Clemens TL, et al. (2010). Mesenchymal stem cells expressing osteogenic and angiogenic factors synergistically enhance bone formation in a mouse model of segmental bone defect. Mol. Ther. 18: 1026-1034.

Liu DP, Li C, Hu L and Wang GX (2010a). Construction of adenovirus vector carrying VEGF121-FLAG and hrGFP-1 and their expressions in mesenchymal stem cells. J. Clin. Rehabilitat. Tissue Engineering Res. 14: 8539-8543.

Liu DP, Wang GX, Hu L and Li Z (2010b). Construction of adenovirus-mediated eukaryotic expression vector coexpressing mutant hypoxia-inducible factor-1 alpha target protein and humanized Renilla reniformis green fluorescent protein reporter molecule under normoxic conditions. J. Clin. Rehabilitat. Tissue Engineering Res. 14: 3787-3792.

Liu D, Hu L, Zhang Z, Li QY, et al. (2013). Construction of human BMP2-IRES-HIF1alphamu adenovirus expression vector and its expression in mesenchymal stem cells. Mol. Med. Rep. 7: 659-663.

Meinel L, Hofmann S, Betz O, Fajardo R, et al. (2006). Osteogenesis by human mesenchymal stem cells cultured on silk biomaterials: comparison of adenovirus mediated gene transfer and protein delivery of BMP-2. Biomaterials 27: 4993-5002.

Menendez MI, Clark DJ, Carlton M, Flanigan DC, et al. (2011). Direct delayed human adenoviral BMP-2 or BMP-6 gene therapy for bone and cartilage regeneration in a pony osteochondral model. Osteoarthritis Cartilage 19: 1066-1075.

Misteli T and Spector DL (1997). Applications of the green fluorescent protein in cell biology and biotechnology. Nat. Biotechnol. 15: 961-964.

Neman J, Hambrecht A, Cadry C and Jandial R (2012a). Stem cell-mediated osteogenesis: therapeutic potential for bone tissue engineering. Biologics 6: 47-57.

Neman J, Hambrecht A, Cadry C, Goodarzi A, et al. (2012b). Clinical efficacy of stem cell mediated osteogenesis and bioceramics for bone tissue engineering. Adv. Exp. Med. Biol. 760: 174-187.

Pérez-Sánchez MJ, Ramírez-Glindon E, Lledó-Gil M, Calvo-Guirado JL, et al. (2010). Biomaterials for bone regeneration. Med. Oral Patol. Oral Cir. Bucal. 15: e517-e522.

Porter JR, Henson A, Ryan S and Popat KC (2009). Biocompatibility and mesenchymal stem cell response to poly (epsilon-caprolactone) nanowire surfaces for orthopedic tissue engineering. Tissue Eng. Part A 15: 2547-2559.

Salunkhe SS, Raiker VA, Rewanwar S, Kotwal P, et al. (2010). Enhanced fluorescent properties of an OmpT site deleted mutant of green fluorescent protein. Microb. Cell Fact. 9: 26.

Xynos ID, Hukkanen MV, Batten JJ, Buttery LD, et al. (2000). Bioglass 45S5 stimulates osteoblast turnover and enhances bone formation In vitro: implications and applications for bone tissue engineering. Calcif. Tissue Int. 67: 321-329. 